\title{
A new and convenient synthesis of 2-arylbenzimidazoles through reaction of benzoylisothiocyanates with ortho-phenylenediamines
}

\author{
Girija S. Singh, ${ }^{* a}$ Tarjeet Singh, ${ }^{b}$ and Ram Lakhan ${ }^{b}$ \\ ${ }^{a}$ Chemistry Department, University of Botswana, Private Bag: 0022, Gaborone, Botswana \\ ${ }^{b}$ Department of Chemistry, Banaras Hindu University, Varanasi-221 005, India \\ E-mail: singhgs@mopipi.ub.bw
}

Dedicated to Prof. Richard R. Schmidt on the occasion of his $78^{\text {th }}$ birthday

DOI: http://dx.doi.org/10.3998/ark.5550190.0014.217

\begin{abstract}
This paper describes the reaction of benzoylisothiocyanates with ortho-phenylenediamines forming the corresponding bisthioureas which undergo cyclization with elimination of thiocyanic acid on refluxing in pyridine leading to the formation of 2-arylbenzimidazoles. The products have been characterized on the basis of satisfactory analytical and spectral (IR, NMR, MS) data, and the mechanism of their formation is proposed.
\end{abstract}

Keywords: Benzimidazoles, phenylenediamines, bisthioureas, cyclodehydration

\section{Introduction}

Benzimidazoles constitute a well-known class of five-membered azaheterocycles possessing different types of biological activity. ${ }^{1}$ These compounds are reported to have anthelmintic, ${ }^{2}$ anticancer, ${ }^{3}$ antihypertensive ${ }^{4}$ and antiulcer activities. ${ }^{5}$ The conventional method for the synthesis of 2-arylbenzimidazoles involves reaction of ortho-phenylenediamines with aromatic acids, nitrile, imidates or ortho-esters under strongly acidic condition at very high temperature. ${ }^{6}$ Recently, the reaction of ortho-phenylenediamine with acid chlorides in the presence of $\mathrm{KF} / \mathrm{Al}_{2} \mathrm{O}_{3}$ as a catalyst has been reported to afford 2-arylbenzimidazoles. ${ }^{7}$ Alternatively, the reactions of ortho-phenylenediamines with aromatic aldehydes in the presence of several oxidizing agents and catalysts such as $\mathrm{MnO}_{2},{ }^{8} \mathrm{PbOAc}_{4},{ }^{9} \mathrm{NaHSO}_{3},{ }^{10} \mathrm{DDQ},{ }^{11}$ thiamine

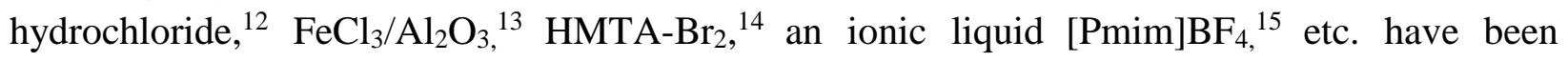
employed for the synthesis of 2-arylbenzimidazoles. A three-component reaction of 2bromo/iodoaniline, aldehydes and sodium azide in the presence of CuCl-TMEDA has been reported to afford 2-arylbenzimidazoles. ${ }^{16}$ Recently, non-conventional techniques such as microwave irradiation $^{17}$ and ultrasonic irradiation ${ }^{18}$ have been employed to achieve easy 
synthesis of 2-arylbenzimidazoles. For example, Joshi and coworkers have reported the synthesis of 2-arylbenzimidazoles by tetrabutylammonium fluoride mediated reaction of phenylene-1,2diamines with aromatic aldehydes in aqueous medium under the influence of ultrasonic irradiation. ${ }^{18}$ Niknam and Fatehi-Raviz reacted phenylene-1,2-diamines with aromatic carboxylic acids in the presence of methylsulfonic acid over neutral alumina by microwave irradiation. ${ }^{19}$

The reactions of benzoylisothiocyanates are of interest to organic chemists from synthetic and mechanistic point of view. ${ }^{20}$ The reactions of these heterocumulenes often depend on the structure of the isothiocyanates. ${ }^{21}$ In the present paper, we report 2:1 molar reactions of bezoylisothiocyanates with 1,2-phenylenediamines resulting into formation of corresponding bisthioureas which undergo cyclization to 2 -arylbenzimidazoles by refluxing in pyridine. There is a report on formation of 2-N-(acyl)aminobenzimidazoles by the reactions of bezoylisothiocyanates with 1,2-phenylenediamines. ${ }^{22}$

\section{Results and Discussion}

A 1:2 molar reaction of 1,2-phenylenediamines $\mathbf{1}$ with benzoylisothiocyanates $\mathbf{2}$ in benzene afforded the corresponding $N, N^{\prime}$-bis(benzoylthiocarbamoyl)-1,2-phenylenediamines $\mathbf{3}$ in almost quantitative yields. The products were characterized on the basis of their analytical and spectral data (Table 1) with reported values. The IR spectra of the products showed a broad band at 3330$3280 \mathrm{~cm}^{-1}$ corresponding to the N-H linkage, a strong band at $1675 \pm 5 \mathrm{~cm}^{-1}$ corresponding to an amide carbonyl absorption, and a strong absorption band around $1150 \mathrm{~cm}^{-1}$ corresponding to the $\mathrm{C}=\mathrm{S}$ bond.

The products 3a-h, after characterization, were dissolved in pyridine and heated at $120{ }^{\circ} \mathrm{C}$ for 15 hours. After distilling off the pyridine and cooling, a solid product was obtained which was washed with water, dried and recrystallized with ethanol. The solid products have been characterized as 2-arylbenzimidazoles $\mathbf{4 a - h}$ on the basis of their analytical and spectral data, and undepressed mixed mp with authentic samples. ${ }^{23}$

The IR spectra of the products 4 showed no absorption in the carbonyl region indicating the removal of both the carbonyl groups in the product. The IR spectra showed a broad band around $3350 \mathrm{~cm}^{-1}$. Similarly, the ${ }^{13} \mathrm{C}$ spectra did not show any downfield signal corresponding to the carbonyl carbons. Instead, a low-field signal at around $\delta 152$ was observed in all products that has been assigned to azomethine carbon. The ${ }^{1} \mathrm{H}$ NMR spectra exhibited a downfield signal corresponding to one proton. This signal was $\mathrm{D}_{2} \mathrm{O}$ exchangeable; so assigned to $\mathrm{NH}$ proton. These significant spectral features of the products led us to characterize these products as 2arylbenzimidazoles. Finally, the authentic samples of $\mathbf{4 a}$ and $\mathbf{4 b}$ were prepared according to a reported method for comparing the $\mathrm{mp}$ and spectral data. The comparison of spectral data and undepressed mixed $\mathrm{mp}$ with the authentic sample confirmed the products as 2arylbenzimidazoles. 
Table 1. Analytical IR spectral data of $N, N^{\prime}$-bis(benzoylthiocarbamoyl)-1,2-phenylenediamines

\begin{tabular}{ccccccc}
\hline No. & $\mathrm{R}^{1}$ & $\mathrm{R}^{2}$ & m.p. $\left({ }^{\circ} \mathrm{C}\right)$ & Yield $(\%)$ & Mol. Formula & IR $\left.\left(\mathrm{KBr}, \mathrm{cm}^{-1}\right)\right)$ \\
\hline 3a & $\mathrm{H}$ & $\mathrm{Ph}$ & $179-180$ & 95 & $\mathrm{C}_{22} \mathrm{H}_{18} \mathrm{~N}_{4} \mathrm{O}_{2} \mathrm{~S}_{2}$ & $3330,1674,1147$ \\
3b & $\mathrm{H}$ & $4-\mathrm{MeOPh}$ & $165-167$ & 98 & $\mathrm{C}_{24} \mathrm{H}_{22} \mathrm{~N}_{4} \mathrm{O}_{4} \mathrm{~S}_{2}$ & $3320,1680,1145$ \\
3c & $\mathrm{H}$ & $4-\mathrm{ClPh}$ & $190-192$ & 90 & $\mathrm{C}_{22} \mathrm{H}_{16} \mathrm{Cl}_{2} \mathrm{~N}_{4} \mathrm{O}_{2} \mathrm{~S}_{2}$ & $3310,1674,1147$ \\
3d & $\mathrm{H}$ & $3-\mathrm{MePh}$ & $159-160$ & 95 & $\mathrm{C}_{24} \mathrm{H}_{22} \mathrm{~N}_{4} \mathrm{O}_{2} \mathrm{~S}_{2}$ & $3330,1674,1147$ \\
3e & $\mathrm{Me}$ & $\mathrm{Ph}$ & $185-187$ & 92 & $\mathrm{C}_{24} \mathrm{H}_{22} \mathrm{~N}_{4} \mathrm{O}_{2} \mathrm{~S}_{2}$ & $3320,1680,1148$ \\
3f & $\mathrm{Me}$ & $3-\mathrm{MePh}$ & $200-201$ & 96 & $\mathrm{C}_{26} \mathrm{H}_{26} \mathrm{~N}_{4} \mathrm{O}_{2} \mathrm{~S}_{2}$ & $3330,1675,1147$ \\
3g & $\mathrm{Me}$ & $4-\mathrm{MeOPh}$ & $169-171$ & 95 & $\mathrm{C}_{26} \mathrm{H}_{26} \mathrm{~N}_{4} \mathrm{O}_{4} \mathrm{~S}_{2}$ & $3320,1678,1145$ \\
3h & $\mathrm{Me}$ & $4-\mathrm{ClPh}$ & $199-200$ & 90 & $\mathrm{C}_{24} \mathrm{H}_{20} \mathrm{Cl}_{2} \mathrm{~N}_{4} \mathrm{O}_{2} \mathrm{~S}_{2}$ & $3320,1680,1145$ \\
\hline
\end{tabular}

*All compounds gave analysis for $\mathrm{C}, \mathrm{H}$, and $\mathrm{N}$ in the range of \pm 0.4 .

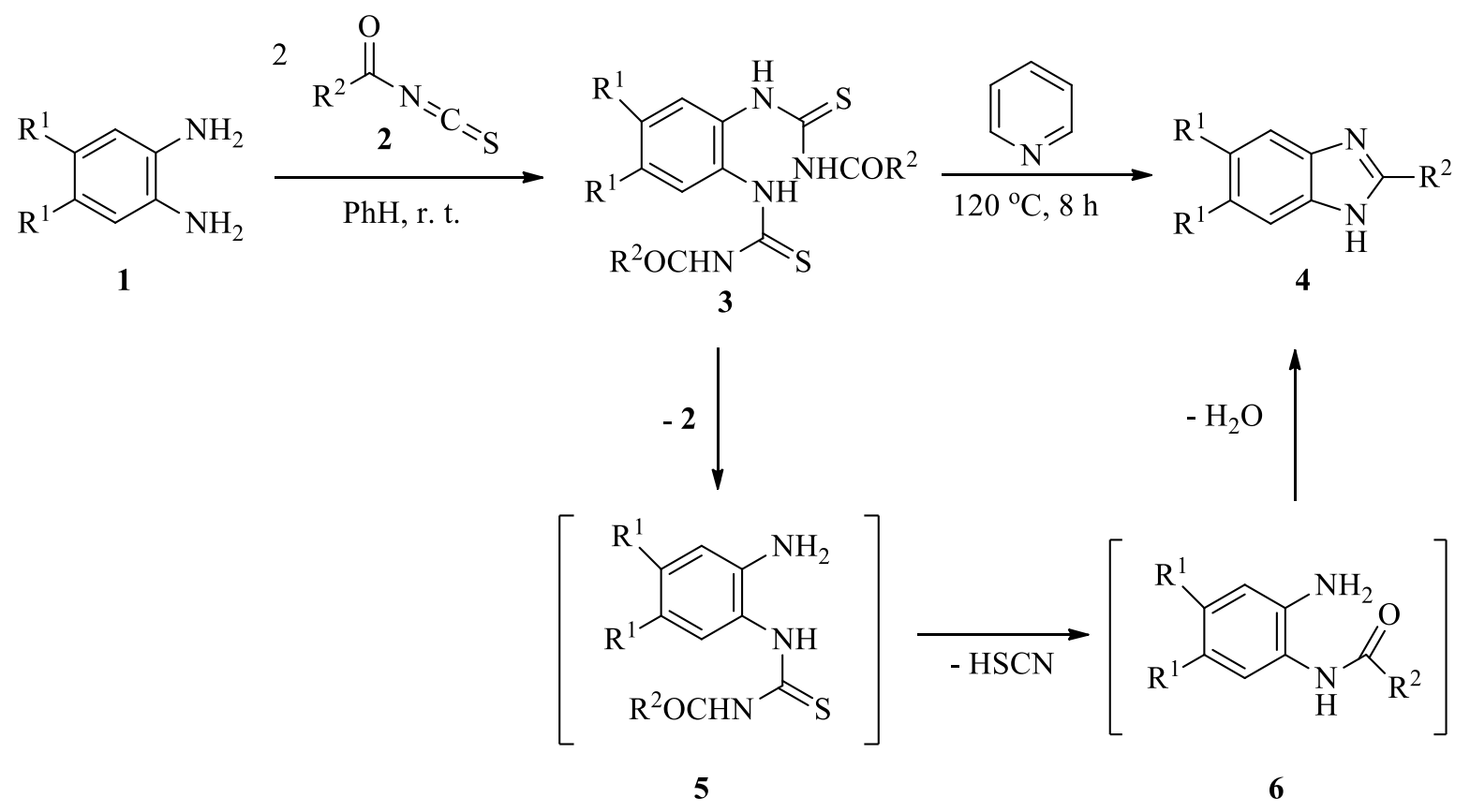

\section{Scheme 1}

The formation of products $\mathbf{3}$ has been explained by usual nucleophilic attack of amines $\mathbf{1}$ on carbon atom of the isothiocyanate linkage in substrates 2 (Scheme 1). On refluxing in pyridine, the removal of one aroylisothiocyanate moiety may lead to the formation of $\mathrm{N}$ (benzoylthiocarbamoyl)-1,2-phenylenediamines 5. The formation of $N$-(benzoylthiocarbamoyl)1,2-phenylenediamines $\mathbf{5}$ from the reaction of amines $\mathbf{1}$ with isothiocyanates $\mathbf{2}$ has been reported previously by refluxing in acetonitrile. ${ }^{22}$ The intermediate product $\mathbf{5}$ may lead to the formation of 2-arylbenzimidazoles 4 by dethiocyanation to intermediate product $\mathbf{6}$ and concomitant cyclodehydration. It is worth mentioning that when intermediate products $\mathbf{5}$ were refluxed in acetonitrile in the presence of DCC, cyclization took place with desulfurization resulting into formation of 2-N-(acyl)aminobenzimidazole indicating the sensitivity of reaction towards 
solvents and cyclodehydrating agents used in the reaction. ${ }^{22}$ The present study demonstrates benzoylisothiocyanates as excellent alternatives to aldehydes and carboxylic acids used in other methods for preparation of 2-arylbenzimidazoles as no catalyst is employed in this case.

\section{Conclusions}

In summary, the paper reports a new and convenient entry to a biologically important heterocyclic compounds, 2-arylbenzimidazoles and demonstrates the dependence of the outcome of the decomposition of $N, N$ '-bis(benzoylthiocarbamoyl)-1,2-phenylenediamines on solvent. This reaction, to the best of our knowledge, is first report on the formation of 2arylbenzimidazoles from these substrates.

\section{Experimental Section}

General. Melting points have been recorded on a Stuart Scientific melting point apparatus and are uncorrected. The IR spectra were recorded on a Perkin-Elmer-781 IR spectrophotometer using $\mathrm{KBr}$ disc of the sample. The ${ }^{1} \mathrm{H}$ and ${ }^{13} \mathrm{C}$ NMR spectra were recorded in a DMSO- $\mathrm{d}_{6}$ solution at $300 \mathrm{MHz}$ and $75.4 \mathrm{MHz}$, respectively, on a Brucker ${ }^{\mathrm{TM}} 300 \mathrm{MHz}$ spectrometer. The mass spectra were recorded on a Finnigan LCQ Deca mass spectrometer by electrospray ionization. The 1,2-phenylenediamines were procured from Aldrich Chemicals and benzoylisothiocynates were prepared by reactions of acid chlorides with potassium thiocyante according to reported methods. ${ }^{24}$

General procedure for the preparation of $N, N^{\prime}$-bis(benzoylthiocarbamoyl)-1,2phenylenediamines (3a-h). Benzoyl isothiocyanates $(10 \mathrm{mmol})$ were added to a solution of 1,2phenylenedimines $(5 \mathrm{mmols})$ in $6 \mathrm{~mL}$ of benzene under vigorous stirring. After complete addition of isothiocyanate, the reaction mixture was cooled in an ice bath to get the solid product in almost quantitative yield. The products were used as such in next step for cyclization reaction.

General procedure for the cyclization of $N, N^{\prime}$-bis(benzoylthiocarbamoyl)-1,2phenylenediamines (3a-h). The product from the last step ( $2 \mathrm{mmol}$ of each) was refluxed in 6 $\mathrm{mL}$ of pyridine on an oil bath for $8 \mathrm{~h}$. After completion of the reaction, pyridine was distilled off and the reaction mixture was allowed to attain the room temperature. A solid product was formed in the flask that was recrystallized with ethanol to get pure products that have been characterized as 2-arylbenzimidazoles on the basis of following analytical and spectral data.

2-Phenyl-1H-benzimidazole (4a). Yield: 75\%; mp 288-290 ${ }^{\circ} \mathrm{C}$ (lit., 290-292 ${ }^{\circ} \mathrm{C}$ ), ${ }^{15} \mathrm{IR}(\mathrm{KBr}$, $\mathrm{cm}^{-1}$ ): 3350, 2995, 1630, 1590; ${ }^{1} \mathrm{H}$ NMR (DMSO-d6): $\delta \mathrm{H} 12.52$ (bs, $\left.1 \mathrm{H}, \mathrm{NH}\right), 7.88$ (d, J $7.8 \mathrm{~Hz}$, $2 \mathrm{H}$, arom.), 7.45-7.35 (m, 5H, Arom.), 7.26-7.12 (m, 2H, arom.); ${ }^{13} \mathrm{C}$ NMR (DMSO-d6): $\delta \mathrm{C}$ 
151.5, 144.0, 135.5, 130.2, 129.4, 128.3, 126.8, 122.0, 121.5. 119.5, 111.8; MS (ESI): $\mathrm{m} / z .194$ $\left(\mathrm{M}^{+}\right)$

2-(4-Methoxyphenyl)-1H-benzimidazole (4b). Yield: 88\%; mp 224-226 ${ }^{\circ} \mathrm{C}$ (lit., $226{ }^{\circ} \mathrm{C}$ ), IR $\left(\mathrm{KBr}, \mathrm{cm}^{-1}\right): 3340,2910,1635,1600 ;{ }^{1} \mathrm{H}$ NMR (DMSO-d $): \delta \mathrm{H} 12.09$ (bs, 1H, NH), 7.85 (d, $J$ $7.6 \mathrm{~Hz}, 2 \mathrm{H}$, arom.), 7.71-7.68 (m, 2H, arom.), 7.52-7.46 (m, 2H, arom.), 7.21 (d, J 8.0 Hz, 2H, arom.), 3.92 (s, $\left.3 \mathrm{H}, \mathrm{OCH}_{3}\right) ;{ }^{13} \mathrm{C}$ NMR (DMSO- $\left.d_{6}\right)$ : $\delta \mathrm{C} 161.0,151.2,144.5,134.5,128.8,123.0$, 122.1, 121.8, 119.2, 115.0, 112.5, 56.5; MS (ESI): $\mathrm{m} / z .224\left(\mathrm{M}^{+}\right)$.

2-(4-Chlorophenyl)-1H-benzimidazole (4c). Yield: 68\%; mp 291-293 ${ }^{\circ} \mathrm{C}$ (lit., 292-293 ${ }^{\circ} \mathrm{C}$ ); IR $\left(\mathrm{KBr}, \mathrm{cm}^{-1}\right): 3350,2920,1630,1600 ;{ }^{1} \mathrm{H}$ NMR (DMSO-d $): \delta \mathrm{H} 12.20$ (bs, $\left.1 \mathrm{H}, \mathrm{NH}\right), 8.10$ (d, $J$ $8.9 \mathrm{~Hz}, 2 \mathrm{H}), 7.45-7.30$ (m, 4H, arom.), 7.15-6.95 (m, 2H, arom.); ${ }^{13} \mathrm{C}$ NMR (DMSO- $\left.d_{6}\right): \delta \mathrm{C}$ $150.5,144.2,135.5,134.9,130.9,129.7,128.8,123.5,122.2,119.5,111.5 ; \mathrm{MS}$ (ESI): $\mathrm{m} / z 228$ $\left(\mathrm{M}^{+}\right)$.

2-(3-Methylphenyl)-1H-benzimidazole (4d). Yield: 82\%; mp 217-219 ${ }^{\circ} \mathrm{C}$ (lit., 217-219 ${ }^{\circ} \mathrm{C}$ ); IR $\left(\mathrm{KBr}, \mathrm{cm}^{-1}\right): 3360,2950,1635,1590 ;{ }^{1} \mathrm{H}$ NMR (DMSO- $\left.d_{6}\right):, \delta \mathrm{H} 12.21$ (bs, $\left.1 \mathrm{H}, \mathrm{NH}\right), 8.12(\mathrm{~d}, J$ 8.1 Hz, 1H, arom.), 7.80 (s, 1H, arom.), 7.59, (d, J 8.0 Hz, 2H, arom.), 7.39-7.19 (M, 4H, arom.), $2.35\left(\mathrm{~s}, 3 \mathrm{H}, \mathrm{CH}_{3}\right),{ }^{13} \mathrm{C}$ NMR (DMSO- $\left.d_{6}\right): \delta \mathrm{C} 151.9,140.4,135.5,131.0,123.0,122.2,119.4$, 111.9, 20.9; MS (ESI): $m / z, 208\left(\mathrm{M}^{+}\right)$.

5,6-Dimethyl-2-phenyl-1H-benzimidazole (4e). Yield: $85 \%$; mp 254-255 ${ }^{\circ} \mathrm{C}$; IR $\left(\mathrm{KBr}, \mathrm{cm}^{-1}\right)$ : 3355, 2910, 1640, 1590; ${ }^{1} \mathrm{H}$ NMR (DMSO-d6): $\delta \mathrm{H} 12.24$ (bs, 1H, NH), 8.28 (d, $8.9 \mathrm{~Hz}, 2 \mathrm{H}$, arom.), 7.51-7.41 (m, 3H, arom.), 7.30 (s, 2H, arom.), 2.49 (s, 6H, two $\mathrm{CH}_{3}$ ); MS (ESI): m/z 222 $\left(\mathrm{M}^{+}\right)$; Anal. Calcd. for $\mathrm{C}_{15} \mathrm{H}_{14} \mathrm{~N}_{2}$ : C 81.05, H 6.35, N 12.60\%. Found: C 80.85, H 6.48, N 12.34\% 5,6-Dimethyl-2-(4-methoxyphenyl)-1H-benzimidazole (4f). Yield: 94\%; mp 263-264 ${ }^{\circ} \mathrm{C}$; IR $\left(\mathrm{KBr}, \mathrm{cm}^{-1}\right): 3360,2920,1630,1585 ;{ }^{1} \mathrm{H}$ NMR (DMSO-d $): \delta \mathrm{H} 12.00$ (bs, 1H, NH), 7.95 (d, $J$ $8.1 \mathrm{~Hz}, 2 \mathrm{H}$, arom), 7.30 (s, 2H, arom), 6.98 (d, 7.9 Hz, 2H, arom), $4.13\left(\mathrm{~s}, 3 \mathrm{H}, \mathrm{OCH}_{3}\right), 2.55(\mathrm{~S}$, $6 \mathrm{H}$, two $\mathrm{CH}_{3}$ ); MS (ESI): $m / z, 252\left(\mathrm{M}^{+}\right)$; Anal. Calcd. for $\mathrm{C}_{16} \mathrm{H}_{16} \mathrm{~N}_{2} \mathrm{O}: \mathrm{C} 76.16, \mathrm{H} \mathrm{6.39}, \mathrm{N}$ $11.10 \%$.. Found: C 76.32, H 6.28, N $11.26 \%$.

5,6-Dimethyl-2-(4-chlorophenyl)-1H-benzimidazole (4g). Yield: 79\%; mp 230-232 ${ }^{\circ} \mathrm{C}$; IR $\left(\mathrm{KBr}, \mathrm{cm}^{-1}\right.$ ): 3360, 2920, 1630, 1600; ${ }^{1} \mathrm{H}$ NMR (DMSO-d6): $\delta \mathrm{H} 12.24$ (bs, 1H, NH), 8.28 (d, 8.9 $\mathrm{Hz}, 2 \mathrm{H}$, arom.), 7.51-7.41 (m, 2H, arom.), 7.30 (s, 2H, arom.), 2.49 (s, 6H, two $\mathrm{CH}_{3}$ ); MS (ESI): $m / z 256\left(\mathrm{M}^{+}\right)$; Anal. Calcd. for $\mathrm{C}_{15} \mathrm{H}_{13} \mathrm{ClN}_{2}$ : C 70.18, H 5.10, N 10.91\%. Found: $\mathrm{C}_{15} \mathrm{H}_{13} \mathrm{ClN}_{2}$ : C 70.28, H 4.98, N $10.78 \%$.

5,6-Dimethyl-2-(3-methylphenyl)-1H-benzimidazole (4h). Yield: 87\%; mp 240-241 ${ }^{\circ} \mathrm{C}$; IR $\left(\mathrm{KBr}, \mathrm{cm}^{-1}\right): 3370,2970,1640,1585 ;{ }^{1} \mathrm{H}$ NMR (DMSO-d6): $\delta \mathrm{H} 12.44$ (bs, $\left.1 \mathrm{H}, \mathrm{NH}\right), 7.90,(\mathrm{~d}, J$ $7.9 \mathrm{~Hz}, 1 \mathrm{H}$, arom.), 7.75 (s, 1H, arom.), 7.32 (s, 2H, arom.), 7.30 (m, 2H, arom.), 2.89 (s, 3H, $\mathrm{CH}_{3}$ ), 2.44 (s, 6H, two $\mathrm{CH}_{3}$ ); $\mathrm{MS}(\mathrm{ESI}): \mathrm{m} / z 236\left(\mathrm{M}^{+}\right)$; Anal. Calcd. for $\mathrm{C}_{16} \mathrm{H}_{16} \mathrm{~N}_{2}$ : C 81.32, $\mathrm{H}$ 6.82, N 11.85\%.. Found: C 81.05, H 6.80, N 11.63\%. 


\section{Acknowledgements}

The authors are thankful to Chemistry Departments of Banaras Hindu University, and University of Botswana, for providing necessary research facilities.

\section{References}

1. For a review on biologically active benzimidazoles: Spasov, A. A.; Yozhista, I. N.;

Bugaeva, L. I.; Anisimova, V. A. Pharm. Chem. J. 1999, 33, 232.

2. Habib, N. S.; Soliman, R.; Ashour, F. A.; El-Taiebi, M. Pharmazie, 1997, 52, 746.

3. Wang, H.; Gupta, R.; Lown, J. W. Anticancer Drug. Dis. 1994, 9, 153.

4. Li, X. C.; Widdop, R. E. Hypertension 1995, 26, 989.

5. Massoomi, F.; Savage, J.; Destache, C. J. J. Pharmacotherapy 1993, 13, 46.

6. Wright, J. B. Chem. Rev. 1951, 48, 396; Middleton, R. W. Wibberley, D. G. J. Heterocycl. Chem. 1980, 17, 1757.

7. Khalili; Bahadori, S.; Sardarin; Reza, A. Monatsh fuer Chem. 2012, 143, 841.

8. Bhatnagar, I.; George, M. V. Tetrahedron 1968, 24, 1293.

9. Stephens, F. F.; Bower, J. D. J. Chem. Soc. 1949, 2971.

10. Austen, S. C.; Kane, J. M. J. Heterocycl. Chem. 2001, 38, 979.

11. Lee, K. J.; Janda, K. D. Can. J. Chem. 2001, 79, 1556.

12. Lei, M.; Ma, L.; Hu, L. Synth. Commun. 2012, 42, 2981.

13. Chen, G.-F.; Dong, X.-Y. E-J. Chem. 2012, 9, 289.

14. Khosravi, K.; Kazemi, S. Chin. Chem. Lett. 2012, 23, 61.

15. Saha, D.; Saha, A.; Ranu, B. C. Green Chem. 2009, 11, 733.

16. Kim, Y.; Kumar, M. R.; Park, N.; Heo, Y.; Lee, S. J. Org. Chem. 2011, 76, 9577.

17. Bourgin, K.; Loupy, A.; Soufiaoui, M. Tetrahedron 1998, 54, 8055; Reddy, G. B.; Rao, V. V. V. N. S. R.; Narsaiahrao, P. S. Synth. Commun. 2002, 32, 2467.

18. Joshi, R. S.; Mandhane, P. G.; Dabhade, S. K.; Gill, C. H. J. Chin. Chem. Soc. 2010, 57, 1227.

19. Niknam, K.; Fatehi-Raviz, A. J. Iran. Chem. Soc. 2007, 4, 438.

20. Insuasty, H.; Estrada, M.; Cortes, E.; Quiroga, J.; Insuasty, B.; Abonia, R.; Nogueras, M.; Cobo, J. Tetrahedron Lett. 2006, 47, 5441; Shaban, M. E. J. Chem. Soc. Pak. 1988, 10, 235; Assy, M. G. Phosphorus, Sulfur, Silicon, Relat. Elem. 1996, 108, 15; Vovk, M. V.; Chubaruk, N. G.; Bol'but, A. V. Russ. J. Org. Chem. 2004, 40, 308.

21. Cambie, R. C.; Davis, P. F.; Rutledge, P. S.; Woodgate, P. D. Aust. J. Chem. 1984, 37, 2073; Palsuledesai, C. C.; Murru, S.; Sahu, S. K.; Patel, B. K. Org. Lett. 2009, 11, 3382. L'Abbe, G.; Francis, A.; Dehaen, W.; Bosman, J. Bull. Soc. Chim. Belg. 1996, 105, 253; Durant, G. J. J. Chem. Soc. C 1967, 952; Coppo, F. T.; Fawzi, M. M. J. Heterocycl. Chem. 1997, 34, 1351. 
22. Kutschy, P.; Ficery, V.; Dzurilla, M. Chem. Pap. 1994, 48, 39; Chem. Abstr. 1994, 121, 108634r.

23. Hein, D. W.; Alheim, R. J.; Leovitt, J. J. J. Am. Chem. Soc. 1957, 79, 427.

24. Ambelang, J. C.; Johnson, T. D. J. Am. Chem. Soc. 1939, 61, 632. 